\title{
eJRIEPS
}

Ejournal de la recherche sur l'intervention en éducation physique et sport

$46 \mid 2020$

Varia

\section{Actualités de la recherche en intervention}

\section{OpenEdition}

Journals

Édition électronique

URL : http://journals.openedition.org/ejrieps/5141

DOI : 10.4000/ejrieps.5141

ISSN : 2105-0821

Éditeur

ELLIADD

\section{Référence électronique}

"Actualités de la recherche en intervention », eJRIEPS [En ligne], 46 | 2020, mis en ligne le 01 avril 2020, consulté le 21 novembre 2020. URL : http://journals.openedition.org/ejrieps/5141 ; DOI : https:// doi.org/10.4000/ejrieps.5141

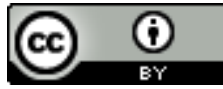

La revue eJRIEPS est mise à disposition selon les termes de la Creative Commons Attribution 4.0 International License. 


\section{Actualités de la recherche en intervention}

\section{Ouvrage: Actes de la 10ème biennale de l'ARIS}

Le sport et l'activité physique sont largement reconnus comme porteurs de valeurs positives profitant à l'ensemble des acteurs du monde socioéducatif, sportif et de santé publique. Cet ouvrage rend compte des travaux menés lors de la 10ème biennale de I'Association pour la Recherche sur l'intervention en Sport (ARIS) organisée à Lille en 2018. Le livre s'organise en quatre axes qui questionnent les interventions selon la perspective des "stratégies responsables d'éducation".

L'intervention dans les pratiques physiques, sportives et artistiques. Responsabilités et stratégies des acteurs, sous la direction de François Potdevin publié en décembre 2019 aux éditions L'Harmattan, collection mouvements des savoirs.

ISBN: 978-2-343-18772-3.

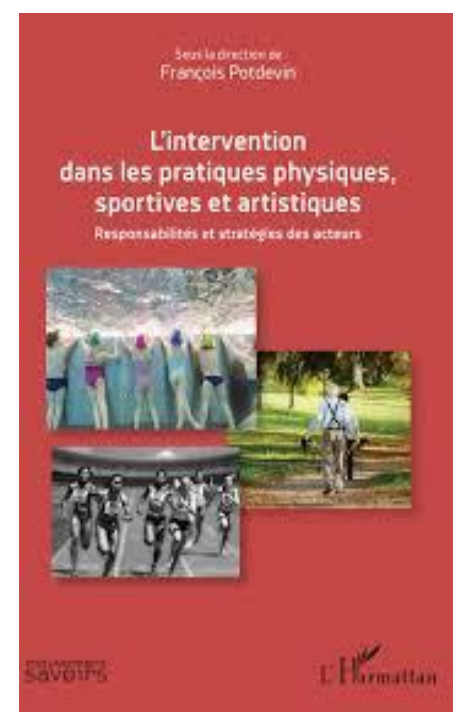

Le Numéro Spécial n`3 Ejrieps, publié en janvier 2020 et coordonné par Ingrid Verscheure et Olivier Vors, vient compléter ces actes de la 10ème Biennale de l'ARIS : https://journals.openedition.org/ejrieps/4206 


\section{Soutenance d'HDR}

Ingrid VERSCHEURE, maîtresse de conférences à l'université de Toulouse 2 Jean Jaurès soutenu son Habilitation à Diriger les Recherches (HDR), intitulée "Genre, Didactique, et Conduite du Changement. Contribution d'un programme de recherche au comparatisme en didactique " soutenue le mardi 11 février 2020, à l'université de Toulouse 2 Jean Jaurès.

\section{Le jury était composé de :}

Chantal AMADE-ESCOT, Professeure des Universités émérite, Sciences de l'Education, Université de Toulouse Jean Jaurès, France (Garante)

Stéphane BRAU-ANTONY, Professeur des Universités, Sciences de l'Education, Université de Reims, France

Isabelle COLLET, Professeure associée, Genre et rapports intersectionnels en formation et éducation, Université de Genève, Suisse

David KIRK, Professor of Education, University of Strathclyde, Ecosse (Rapporteur) Florence LIGOZAT, Professeure associée, Didactique comparée, Université de Genève, Suisse (Rapporteure)

Jean-François MARCEL, Professeur des Universités, Sciences de l'Education, Université Toulouse 2 Jean Jaurès, France (Rapporteur - Président du jury)

\section{Quelques éléments sur le contenu de la note de synthèse}

Depuis la thèse (2005), je mène mes recherches à partir d'études observationnelles dans les classes clairement conduites sous couvert d'un cadre théorique didactique. Cela me permet de tenir ensemble le point de vue performatif que je développe sur le genre et le point de vue didactique dans ce qu'il est spécifique des savoirs de l'École. Mes recherches se situent à l'échelle d'analyse relevant d'une approche microdidactique (c'est à dire propre aux situations de classe) et se sont attachées à rendre compte de la « co-construction des savoirs et du Genre dans la classe ». Elles montrent, par l'analyse de sa dynamique évolutive au fil des situations et des séances, ses effets sur les trajectoires didactiques des élèves filles et garçons. Plus récemment, mes travaux confirment que l'analyse compréhensive des pratiques didactiques, 


\section{eJRIEPS 46 avril 2020}

permet d'identifier de possibles leviers de changement intégrant la problématique du Genre.

La note de synthèse est structurée en trois grands chapitres.

Le premier chapitre, intitulé "La fabrication des inégalités scolaires selon le sexe : nécessité d'une (re)problématisation didactique ", vise à rendre compte de la cohérence de mon travail de chercheure. Après l'avoir situé dans les contextes scientifiques et les cultures de recherche, relatives au genre et à la didactique, j'en décris les évolutions au fil de la vingtaine d'années qui se sont écoulées entre mon « entrée » en recherche et ce moment particulier que constitue la rédaction d'une HDR. Le deuxième chapitre propose un retour réflexif sur un domaine de recherche en voie de constitution : "Genre et didactique ». Dans cette rétrospective, je reviens de façon thématique sur mon parcours scientifique et sur les étapes qui constituent les prémices du programme de recherche que je souhaite poursuivre et développer. J'en pointe les avancées, les zones d'ombres à la fois théoriques et méthodologiques, mais aussi les perspectives ouvertes par les résultats produits.

Le troisième chapitre présente le programme de recherche "Genre et didactique ». La première section est l'occasion de préciser ma posture de chercheure et sa singularité au regard des différentes approches du courant des recherches participatives en éducation. Dans la deuxième section, je développe les fondements théoriques et méthodologiques du programme de recherche à m'appuyant sur les avancées et le dépassement des zones d'ombres identifiées lors du chapitre précédent. Je reviens sur la construction progressive du concept de positionnement de genre épistémique en lien avec le contrat didactique différentiel. Ensuite, je montre en quoi le positionnement de genre épistémique, en tant que dimension de l'épistémologie pratique des acteurs et actrices de la relation didactique, impose à la chercheure de symétriser - dans l'enquête - l'investigation des épistémologies pratiques du professeur et des élèves. Je discute enfin des questions de méthode pour mener des recherches didactiques prenant en compte le Genre. La dernière section de ce troisième chapitre conclut la note de synthèse en synthétisant les apports du programme Genre et Didactique, notamment sa contribution au projet comparatiste en didactique. 


\section{Soutenance de thèse}

Cathy BONBONNE a présenté sa thèse le 9 décembre 2019 à l'Université des Antilles en vue de l'obtention du grade de docteur en sciences de l'éducation. Cette thèse est intitulée "Usages sociaux et didactiques de l'eau en contexte(s) scolaire(s) guadeloupéen(s). Analyse selon une approche socio-didactique ».

\section{Le jury était composé de :}

Frédéric ANCIAUX, Maître de conférences HDR en sciences de l'éducation, CRREF, Université des Antilles, France.

Fabienne BRIERE, Maître de conférences HDR en sciences de l'éducation, ADEF, Université Aix-Marseille, France (Rapporteure).

Antoine DELCROIX, Professeur des universités, CRREF, Université des Antilles, France.

Marie-Paule POGGI, Maître de conférences HDR en sciences de l'Education, CRREF, Université des Antilles, France

Frédéric SAUJAT, Professeur des Universités, ADEF, Université Aix-Marseille, France.

Nathalie WALLIAN, Professeur des Universités, ICARE, Université de La Réunion (Rapporteure).

\section{Résumé}

Notre recherche interprétative et compréhensive s'intéresse aux interactions entre usages sociaux et didactiques de l'eau des enseignants et des élèves du cycle 3 en situation d'enseignement-apprentissage de l'APSA natation en contexte(s) scolaire(s) guadeloupéen(s). Elle croise la sociologie dispositionnaliste et contextualiste (Lahire, 2012) et la didactique, notamment les savoirs savants basés sur la Théorie de l'Action Conjointe en Didactique (TACD), les savoirs professionnels (Gal, 1993) et institutionnels. Nous observons deux niveaux de la contextualisation didactique (la contextualisation pédagogique et sociocognitive) (Delcroix et al., 2013). Notre problématique est la suivante: quelles conditions socio-didactiques favorisent la construction, l'actualisation ou l'inhibition des usages sociaux de l'eau des élèves du premier degré ? 


\section{eJRIEPS 46 avril 2020}

Nous émettons deux hypothèses : 1) les usages sociaux de l'eau sont engendrés par des dispositions incorporées qui se construisent, s'intériorisent et s'actualisent dans les pratiques (situations didactiques) de différentes façons selon les acteurs et peuvent se classer par typologie ; 2) le contexte didactique est un contexte socialisateur ou actualisateur ou inhibiteur des usages sociaux de l'eau des élèves.

Les usages sociaux de l'eau sont des formes de relation à l'eau plus ou moins stables, récurrentes qui se caractérisent par : des représentations symboliques de l'eau, des sensibilités vis-à-vis de l'eau et des dispositions à agir dans l'eau. Les usages didactiques de l'eau sont des pratiques enseignantes qui visent des apprentissages passant par le corps dans l'élément eau. Les connaissances construites sur les actions des acteurs placés en contexte réel où ses actions se déroulent, avec en arrière-plan, des éléments contextuels d'ordre historique, social et culturel, propres à la Guadeloupe, nécessitent l'utilisation couplée de méthodes d'analyse qualitative et quantitative.

Une première étude méso et micro-didactique qualitative concerne quatre enseignants du cycle 3 et se base sur des séances filmées de natation et sur le contenu d'entretiens semi-directifs ante et post vidéo. Nous mettons en évidence le poids du rapport expérientiel à I'APSA natation des enseignants, différentes logiques d'action des élèves induites par les usages didactiques de l'eau des enseignants (construction de nouvelles dispositions à agir dans l'eau ou actualisation ou inhibition des dispositions incorporées) et quatre types d'effets de contextes attribuables à la contextualisation pédagogique (empêchement partiel d'accès au savoir, désemboîtement des savoirs, bifurcations didactiques stables ou instables et focalisation secondaire). Une deuxième étude macrosociologique quantitative concerne 90 d'élèves du cycle 3 et renseignent sur leurs usages sociaux de l'eau. Ces derniers partagent des représentations symboliques variées de l'eau et attribuent à l'APSA natation des valeurs différentes de celles de l'école. Une troisième étude ethnosociologique qualitative se base sur des trajectoires de vie de vingt guadeloupéens et montre la force de la socialisation familiale dans la construction et l'actualisation des usages sociaux de l'eau de ces derniers.

Mots-clés : contextualisation didactique - usages sociaux de l'eau - usages didactiques de l'eau 


\section{Soutenance de thèse}

Claire DEBARS a présenté sa thèse le mercredi 12 février 2020, à l'Université de Toulouse en vue de l'obtention du grade de docteur en sciences de l'éducation. Cette thèse est intitulée "Analyse didactique des pratiques d'enseignement et d'étude selon le genre en contexte d'éducation prioritaire. Études de cas en éducation physique et sportive lors de deux cycles de handball en collège REP+ ».

\section{Le jury était composé de :}

Chantal AMADE-ESCOT, Professeure des universités émérite en sciences de l'éducation, Université de Toulouse Jean-Jaurès, co-directrice de thèse ;

Stéphane BRAU-ANTONY, Professeur des universités en sciences de l'éducation, Université de Reims, rapporteur et président du jury ;

Lucile LAFONT, professeure des universités émérite en STAPS, Université de Bordeaux, examinatrice ;

Marie-Paule POGGI, Maîtresse de conférences en sciences de l'éducation Habilitée à diriger les recherches, Université des Antilles, rapporteure ;

Patrice VENTURINI, Professeur des Universités émérite en sciences de l'éducation, Université de Toulouse Jean Jaurès, examinateur.

Ingrid VERSCHEURE, Maîtresse de conférences en sciences de l'éducation Habilitée à diriger les recherches, Université de Toulouse Jean-Jaurés, co-directrice de thèse

\section{Résumé}

La thèse vise à analyser les pratiques d'éducation physique et sportive en réseau d'éducation prioritaire REP+ en collège à partir de la focale du genre dans l'action didactique conjointe. La méthodologie, de type qualitatif, relève d'études de cas. Elle mobilise deux échelles d'analyse. A un niveau macrodidactique, nous recomposons pour chaque séance et au niveau du cycle l'avancée des savoirs ainsi que les variations du type d'interactions verbales adressées aux filles et aux garçons de l'ensemble de la classe. Cette échelle permet de caractériser la direction d'étude des deux enseignantes (une femme et un homme) selon le sexe. A un niveau microdidactique, nous étudions plus particulièrement, dans chaque site, quatre élèves, 


\section{eJRIEPS 46 avril 2020}

filles et garçons de niveaux contrastés en termes d'excellence scolaire et d'habileté en EPS, lors de certains épisodes remarquables.

La mobilisation du concept de positionnement de genre épistémique permet d'éclairer les processus différentiels à l'œuvre. A cette échelle d'analyse, les résultats mettent en évidence l'articulation du positionnement de genre du professeur et des élèves et son rôle dans l'actualisation des trajectoires didactiques des élèves lors du cycle. II ressort que les phénomènes différentiels selon le genre se redoublent en raison des difficultés à maintenir l'épaisseur des enjeux de savoirs lors des transactions. La recherche met en évidence une pluralité de facteurs intervenant dans ces processus en pointant l'intérêt d'une démarche inspirée de l'intersectionnalité pour rendre compte des inégalités de réussite en EPS des filles et des garçons en éducation prioritaire.

\section{Mots-clés :}

Didactique, Éducation Physique et Sportive, Action didactique conjointe, Genre, Réseau d'Éducation Prioritaire, Handball.

\section{Soutenance de thèse}

Clément LLENA a présenté sa thèse le vendredi 22 novembre 2019, à l'Université de Bordeaux en vue de l'obtention du grade de docteur en sciences et techniques des activités physiques et sportives. Cette thèse est intitulée "Enseigner ce que l'on est : quand la concordance de valeurs rime avec bien-être au travail. Le cas des enseignants d'EPS de l'académie de Lille ».

\section{Le jury était composé de :}

Ghislain CARLIER, Professeur des universités émérite, Université catholique de Louvain, rapporteur ;

Jean-François DESBIENS, Professeur des universités, Université de Sherbrooke, rapporteur ;

Isabelle JOING, Maître de conférences, Université de Lille, co-encadrante de thèse ; Jacques MIKULOVIC, Professeur des universités, Université de Bordeaux, directeur de thèse ; 


\section{eJRIEPS 46 avril 2020}

Nicole RASCLE, Professeure des universités, Université de Bordeaux, présidente du jury ;

Carole SÈVE, Professeure des universités, Inspectrice Générale de l'Éducation Nationale (groupe EPS), examinatrice.

\section{Résumé}

L'enseignant est, dans son exercice professionnel, guidé par des motivations personnelles qui se nourrissent de ses propres valeurs. Celles-ci se traduisent par des comportements, des discours et des attitudes et in fine, caractérisent un style pédagogique. Leur importance est relative et crée une hiérarchie pouvant être différente d'un enseignant à l'autre. Dès lors, se pose la question de savoir si certaines valeurs permettraient d'être davantage en bien-être au travail. Plus encore, le fait d'agir en cohérence par rapport à ses valeurs dans son enseignement serait-il un facteur propice à ce bien-être ?

L'objectif de la thèse consiste à étudier les relations entre le bien-être au travail et les valeurs des enseignants d'Éducation Physique et Sportive (EPS).

En s'inscrivant dans le cadre théorique des valeurs de base de la personne (Schwartz, 1992), un outil de mesure a été conçu pour examiner les valeurs des enseignants d'EPS dans le contexte particulier de l'enseignement de l'EPS avec 599 enseignants d'EPS. Ensuite, le travail a été mené en deux temps. En premier lieu, 396 enseignants d'EPS de l'académie de Lille ont complété un questionnaire permettant d'identifier leur système de valeurs général, leur système de valeurs opérationnalisé en EPS et leur niveau de bien-être subjectif au travail.

Les résultats issus des analyses statistiques multifactorielles montrent que les valeurs sont déterminantes pour expliquer le bien-être au travail. Ainsi, ils révèlent que les valeurs d'ouverture au changement et de dépassement de soi sont plus vertueuses que les valeurs de continuité pour le bien-être des enseignants d'EPS. Si la nature des valeurs permet, en partie, d'expliquer le bien-être au travail, le fait d'agir en accord avec son système général de valeurs est un facteur plus déterminant. Ainsi, la concordance entre ses valeurs et ses pratiques professionnelles apparaît comme un objectif prioritaire pour améliorer le bien-être au travail. De plus, les résultats permettent d'identifier quatre profils caractéristiques d'enseignants selon leurs 


\section{eJRIEPS 46 avril 2020}

systèmes de valeurs et leur niveau de bien-être : les harmonieux, les compositeurs, les désaccordés et les sans-partitions.

Parallèlement à ces enquêtes, douze entretiens semi-directifs ont été menés auprès d'enseignants d'EPS typiques des profils identifiés (trois par profil).

Les résultats issus de l'analyse des entretiens permettent non seulement d'affiner la compréhension des profils d'enseignants d'EPS mais également de mieux comprendre le lien entre leurs systèmes de valeurs et leur niveau de bien-être au travail. Par ailleurs, les résultats révèlent que le partage de valeurs avec ses pairs est un facteur médiateur du bien-être au travail des enseignants d'EPS.

En conclusion, ce travail de recherche basé sur une méthodologie mixte permet d'amorcer une réflexion pédagogique et didactique autour de l'importance des valeurs et de leur concordance dans l'enseignement. II soulève également l'importance de clarifier collectivement les valeurs au sein des équipes pédagogiques. Une réflexion et un travail sur ces deux aspects devraient permettre d'améliorer le bien-être au travail des enseignants.

\section{Mots-clés :}

Domaines de valeurs, Contexte personnel/professionnel, Système de valeurs, Valeurs opérationnalisées, Enseignement, Bien-être, Éducation Physique et Sportive. 